\title{
Collisional excitation of hydrogen and the determination of the primordial helium abundance from $\mathrm{H}$ ॥ regions
}

\author{
G. Stasińska ${ }^{1}$ and Y. Izotov ${ }^{2}$ \\ 1 DAEC, Observatoire de Paris-Meudon, 92195 Meudon Cedex, France \\ 2 Main Astronomical Observatory, Ukrainian National Academy of Sciences, Kyiv 03680, Ukraine \\ e-mail: izotov@mao.kiev.ua
}

Received 11 July 2001 / Accepted 10 September 2001

\begin{abstract}
This paper investigates the effect of collisional enhancement of the hydrogen lines on the derivation of the helium abundances in low metallicity H II regions. For this, we have constructed a grid of photoionization models relevant for the analysis of giant $\mathrm{H}$ II regions in blue compact galaxies. We show that the effect of collisional excitation on the $\mathrm{H} \alpha / \mathrm{H} \beta$ ratio can be quite important (up to $8 \%$ or more). The impact of this effect on the determination of the helium mass fraction has been tracked on four low-metallicity blue compact galaxies for which Keck spectra are available and which are among the best objects for the quest for the pregalactic helium abundance. We find that taking into account the effects of collisional excitation of hydrogen results in an upward correction of the helium mass fraction $Y$ by up to $5 \%$. However, combined with other systematic effects usually not considered in the determination of the helium abundance in low-metallicity galaxies, the resulting uncertainty should be much less.
\end{abstract}

Key words. galaxies: abundances - galaxies: ISM - galaxies: starburst - ISM: H II regions

\section{Introduction}

Tests of the big bang models and the determination of cosmological parameters require an accurate determination of the relative abundances of the light isotopes produced during the big bang (Steigman et al. 1977; Walker et al. 1991; Sarkar 1996). The abundance of primordial ${ }^{4} \mathrm{He}$ is mostly derived from the helium abundances in metal-poor extragalactic H II regions (Kunth \& Sargent 1983; Pagel et al. 1992; Izotov \& Thuan 1998) although other nebulae such as $\mathrm{H}$ II regions in the Magellanic Clouds (Peimbert et al. 2000) or planetary nebulae in the Galactic halo (Peimbert 1983, 1989, Clegg 1989) have also occasionally been used. This is a challenging task, since the required accuracy is on the one percent level.

In order to achieve such a goal, excellent quality observations are obviously required together with an extremely reliable reduction procedure. With the advent of very large telescopes and the progress in detectors, recent years have produced a large amount of data suitable for such an enterprise (Izotov et al. 1997; Izotov \& Thuan 1998; Izotov et al. 1999). As regards the interpretation of the observed line intensities in terms of abundance ratios, many problems occur and still remain to be overcome. For example, the atomic data relevant for the helium line emissivities have only recently reached a state compatible

Send offprint requests to: G. Stasińska,

e-mail: grazyna.stasinska@obspm.fr with the demand (Benjamin et al. 1999). Among the problems to be solved for the determination of the helium abundance in one nebula, and towards which various groups of researchers have had different approaches, are: the stellar absorption lines underlying the nebular emission lines, the reddening correction, the distribution of the electron temperature inside the nebulae, the density structure and its effect on the line emissivities, the optical depth in the lines, the ionization correction to derive the $\mathrm{He} / \mathrm{H}$ ratio from the measured $\mathrm{He}^{+} / \mathrm{H}^{+}$ratio. The next step is to derive the pregalactic helium abundance from the measurement of the helium abundance in a sample of galaxies, by extrapolating the results towards zero metallicity. These problems have been extensively discussed in a long list of papers including Peimbert \& Torres-Peimbert (1974, 1976), Stasińska (1980), Rayo et al. (1982), Kunth \& Sargent (1983), Davidson \& Kinman (1985), Dinerstein \& Shields (1986), Pagel et al. (1986, 1992), Campbell (1992), Skillman \& Kennicutt (1993), Balbes et al. (1993), Mathews et al. (1993), Olive \& Steigman (1995), Sasselov \& Goldwirth (1995), Olive et al. (1997), Steigman et al. (1997), Izotov \& Thuan (1998), Izotov et al. (1997, 1999), Armour et al. (1999), Ballantyne et al. (2000), Viegas et al. (2000), Peimbert et al. (2000, 2001), Olive \& Skillman (2001), Sauer \& Jedamzik (2001).

The present-day situation is that different groups reach different estimates of the pregalactic helium abundance, mutually exclusive within the quoted error bars. For 
example, Olive et al. (1997) give $Y_{\mathrm{p}}=0.234 \pm 0.002$, while Izotov \& Thuan (1998) give $Y_{\mathrm{p}}=0.244 \pm 0.002$. These two estimates lead to very different cosmological implications, as discussed by Izotov et al. (1999).

The effect on the pregalactic helium abundance determination arising from deviation from case B theory for the hydrogen lines has been relatively little discussed so far. The effect of finite optical depth and dust on $\mathrm{H} \beta$ emissivity have been discussed by Cota \& Ferland (1988) and Hummer \& Storey (1992). The effect of collisional excitation of the hydrogen lines by thermal electrons has been pointed out by Davidson \& Kinman (1985) and discussed for the case of I Zw 18 by Sasselov \& Goldwirth (1995) and Stasińska \& Schaerer (1999). Stasińska (2001) has shown that this effect can lead to an underestimation of the He I $5876 / \mathrm{H} \beta$ ratio by as much as $10 \%$ in hot planetary nebulae, independent of the actual reddening of the object.

The purpose of the present paper is to systematically explore the effects of collisional excitation of the hydrogen lines on the helium abundance determinations in metalpoor giant H II regions. For this, we use a grid of photoionization models of giant $\mathrm{H}$ II regions and consider them in the light of the spectral properties of a sample of blue compact H II galaxies. This allows us to obtain a realistic estimate of the effect of collisional excitation by thermal electrons both on $\mathrm{H} \alpha$ and on $\mathrm{H} \beta$. We then concentrate on a selection of blue compact galaxies with particularly good quality spectroscopic data, and track the effect of collisional excitation - at the level predicted by the models - on the derived helium abundance for these objects.

\section{The photoionization models}

The photoionization models of giant $\mathrm{H}$ II regions are constructed using the procedure described in Stasińska et al. (2001, hereinafter SSL2001) to which we refer for details. Briefly, we consider coeval stellar clusters of given initial mass $M_{\star}$ that ionize the surrounding gas, supposed to be of same chemical composition as the stars. We assume instantaneous bursts with a Salpeter initial mass function and an upper stellar limit $M_{\text {up }}=120 M_{\odot}$. The radiation field is provided by the evolutionary synthesis models of Schaerer \& Vacca (1998) based on the nonrotating Geneva stellar evolution models, with the high mass-loss tracks of Meynet et al. (1994). The spectral energy distributions for massive main-sequence stars are those given by the CoStar models (Schaerer \& de Koter 1997). The pure He models of Schmutz et al. (1992) are used for Wolf-Rayet stars. The ionization and temperature structure of the nebula is computed with the photoionization code PHOTO, as described in Stasińska \& Leitherer (1996, hereinafter SL96). We assume that the nebulae are spherical and radiation-bounded, with uniform density $n=10 \mathrm{~cm}^{-3}$ and filling factor $\epsilon=1$ and that all the stars are located at the center. As in our previous papers (SL96 and SSL2001) and in all the papers based on static photoionization models, our models do not consider the possible pollution of the nebulae by stellar ejecta during the course of the evolution of the star clusters. While this phenomenon may have important consequences, it is not really relevant to the problem under study here.

The grid used in the present study is composed of models with metallicities $Z$ of $0.2,0.05$ and 0.02 times the solar metallicity (using the prescription of McGaugh (1991) for the relative abundances of the elements). As is known (e.g. Shields 1986) the emission line spectrum of photoionized nebulae is essentially dependent on the spectral energy distribution of the ionizing radiation field, on the metallicity and on the ionization parameter $U$. For a constant density sphere $U$ is equal to $A\left(Q\left(\mathrm{H}^{0}\right) n \epsilon^{2}\right)^{1 / 3}$, where $Q\left(\mathrm{H}^{0}\right)$ is the total number of $\mathrm{H}$ Lyman continuum photons emitted by the ionizing source (proportional to the mass of the star cluster) and $A$ is approximately given by $A \approx 2.8 \times 10^{-20}\left(10^{4} / T_{\mathrm{e}}\right)^{2 / 3}$, where $T_{\mathrm{e}}$ is the electron temperature. At a given chemical composition, any combination of $Q\left(\mathrm{H}^{0}\right), n$ and $\epsilon$ giving the same value of $U$ will result in the same emission line spectrum (at least when the densities are sufficiently low that cooling is not affected by collisional deexcitation of forbidden lines, which is the case of the objects under study in this paper). Three different initial masses for the star clusters are considered: $M_{\star}=10^{3}, 10^{6}$, and $10^{9} M_{\odot}$ (assuming a lower IMF mass cut-off of $\left.M_{\text {low }}=0.8 M_{\odot}\right)$. With such a grid, we reasonably cover the range of metallicities and ionization parameters relevant to the objects we are interested in.

For each model, we derive the electron temperature $T_{\text {[O III }] 4363 / 5007}$ from the computed [O III] $\lambda 43363 /$ $\left[\begin{array}{ll}\mathrm{O} & \mathrm{III}\end{array} \lambda 5007\right.$ ratio $^{1}$, using the same atomic data as in the photoionization code. Figure 1 presents the sequences of models in the $\left.T_{[\mathrm{O}} \mathrm{III}\right] 4363 / 5007$ versus $\left[\begin{array}{ll}\mathrm{O} & \mathrm{III}\end{array}\right] \lambda 5007 /\left[\begin{array}{ll}\mathrm{O} & \mathrm{II}\end{array}\right] \lambda 3727$ plane (left) and in the $T_{\text {[O III }] 4363 / 5007}$ versus $[\mathrm{O}$ III $] \lambda 5007 / \mathrm{H} \beta$ plane (right). The top panels correspond to a total initial stellar mass of $M_{\star}=10^{3} M_{\odot}$, the middle ones to $M_{\star}=10^{6} M_{\odot}$ and the bottom ones to $M_{\star}=10^{9} M_{\odot}$. Symbols mark the epoch by time steps of $10^{6} \mathrm{yr}$, starting from an age of $10^{4} \mathrm{yr}$. Series with $Z=0.02 Z_{\odot}$ are represented by circles, series with $Z=0.05 Z_{\odot}$ by squares, and series with $Z=0.2 Z \odot$ by triangles. The time evolution is from the right to the left in these plots, since the ionization parameter decreases due to gradual disappearance of the most massive stars. This provokes a softening of the ionizing radiation field, which induces a decrease in the electron temperature. As expected, the highest temperatures are found in the models with lowest metallicities (cooling by collisional excitation of forbidden lines becomes less important) and highest ionization parameters (cooling by collisional excitation of H Lyman $\alpha$ becomes less important).

In the next section, we discuss the relevance of this grid of models for the problem we are investigating in the present paper.

1 Throughout the paper, [OIII] $\lambda 5007$ refers to the intensity of the line at $5007 \AA$, not to the sum of the lines [O III] $\lambda 4959$ and $\lambda 5007$. 

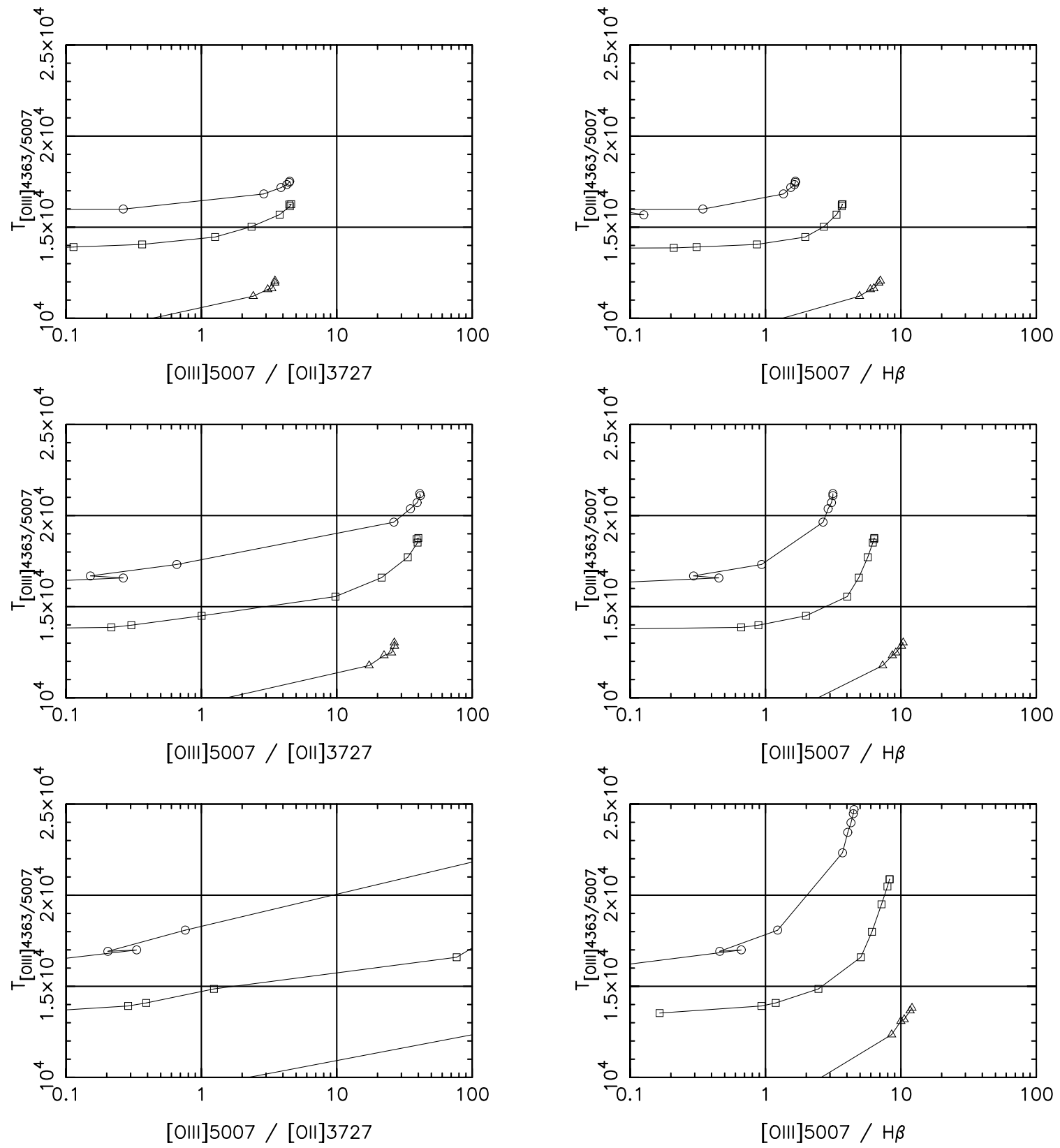

Fig. 1. Sequences of photoionization models for instantaneous bursts of various metallicities. The metallicity is indicated by the following symbols: circle for $Z / Z_{\odot}=0.02$, square for $Z / Z_{\odot}=0.05$, triangle for $Z / Z_{\odot}=0.2$. The symbols mark time steps of 1 Myr. The models shown in the top panels have $M_{\star}=10^{3} M_{\odot}$, those in the middle panels have $M_{\star}=10^{6} M_{\odot}$, those in the bottom panels have $M_{\star}=10^{9} M_{\odot}$ (see text). The ordinate represents the temperature indicated by the [O III $] \lambda 4363 /[\mathrm{O} \mathrm{III}] \lambda 5007 \mathrm{line}$ ratio. In the left panels it is shown as a function of $[\mathrm{O} \mathrm{III}] \lambda 5007 /[\mathrm{O} \mathrm{II}] \lambda 3727$, in the right panels as a function of $[\mathrm{O} \mathrm{III}] \lambda 5007 / \mathrm{H} \beta$.

\section{The sample of blue compact galaxies confronted to the model grid}

Since ab initio models of nebulae photoionized by evolving starbursts do not necessarily well represent the observed properties of giant $\mathrm{H}$ II regions (see discussion in SSL2001), we have to use them in the light of observed properties of H II galaxies. For this, we consider a sample of blue compact galaxies observed since 1994 by Izotov and coworkers. In addition to the 69 objects considered in the study by SSL2001, it contains 64 new objects observed with different telescopes, including Keck, VLT, MMT, 4 m and $2.1 \mathrm{~m}$ KPNO (Izotov et al. 2001a,b; Guseva et al. 2001; Papaderos et al. 2001). The new observations were reduced in the same way as those considered by SSL2001. In all cases, the line intensities have been corrected for reddening by using a procedure described in Izotov et al. (1994) in which the reddening constant and stellar absorption in the Balmer lines are determined simultaneously. Figure 2 shows the same observational diagrams as Fig. 2 of SSL2001 for the new sample of 133 objects. Exactly the same trends are seen (with a slightly higher dispersion due to the fact that some of the new data are of lower quality than those of the sample considered by SSL2001). 



Fig. 2. Our observational sample in various diagrams relating emission line ratios and $\mathrm{H} \beta$ equivalent width. On the upper right of each panel is given the total number of the objects appearing in the diagram. The behaviour of the present sample in these diagrams is very similar to the behaviour of the smaller sample shown by Stasińska et al. (2001) in their Fig. 2.

Therefore, we are dealing with the same population of objects. Hence the conclusions of SSL2001 still apply: the sample is mostly composed of objects whose ionization is produced by a starburst of age younger than about $5 \mathrm{Myr}$. In the following, we will then restrict our considerations to models corresponding to such ages.

Figure 3 shows the observed sample in the $\left.T_{[\mathrm{O}} \mathrm{III}\right] 4363 / 5007$ versus $[\mathrm{O}$ III $] \lambda 5007 /[\mathrm{O}$ II $] \lambda 3727$ plane (left) and in the $\left.T_{[\mathrm{O}} \mathrm{III}\right] 4363 / 5007$ versus $[\mathrm{O}$ III $] \lambda 5007 / \mathrm{H} \beta$ plane (right). By comparing with Fig. 1, we see that while the models with the highest $T_{[\mathrm{O} \text { III }] 4363 / 5007}$ well reproduce the $[\mathrm{O}$ III $] \lambda 5007 / \mathrm{H} \beta$ ratios, they have $[\mathrm{O}$ III $] \lambda 5007 /[\mathrm{O}$ II $] \lambda 3727$ ratios much larger than observed in objects of similar values of $T_{[\mathrm{O}}$ III] $4363 / 5007$. For the range of observed values of $[\mathrm{O}$ III $] \lambda 5007 /[\mathrm{O}$ II $] \lambda 3727$, it is impossible to obtain models with $T_{[\mathrm{O} \text { III] } 4363 / 5007}$ around $20000 \mathrm{~K}$. One possible way to increase the $[\mathrm{O}$ II] line intensity is to consider composite models, in which part of the emission comes from higher density filaments. However, it was impossible to find a prescription for the composite models to reproduce at the same time $[\mathrm{O}$ III $] \lambda 4363 /[\mathrm{O}$ III $] \lambda 5007$, $\left[\begin{array}{ll}\mathrm{O} & \mathrm{III}\end{array}\right] \lambda 5007 /\left[\begin{array}{l}\mathrm{O} \\ \mathrm{II}\end{array}\right] \lambda 3727$ and the electron density sen-

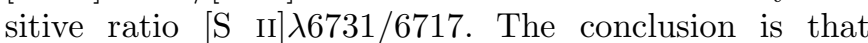
simple photoionization models are unable to reproduce the high $[\mathrm{O}$ III $] \lambda 4363 /[\mathrm{O}$ III $] \lambda 5007$ ratios that are observed in quite a number of giant $\mathrm{H}$ II regions. A similar conclusion has been reached from tailored model fitting of various giant H II regions (Campbell 1988; 

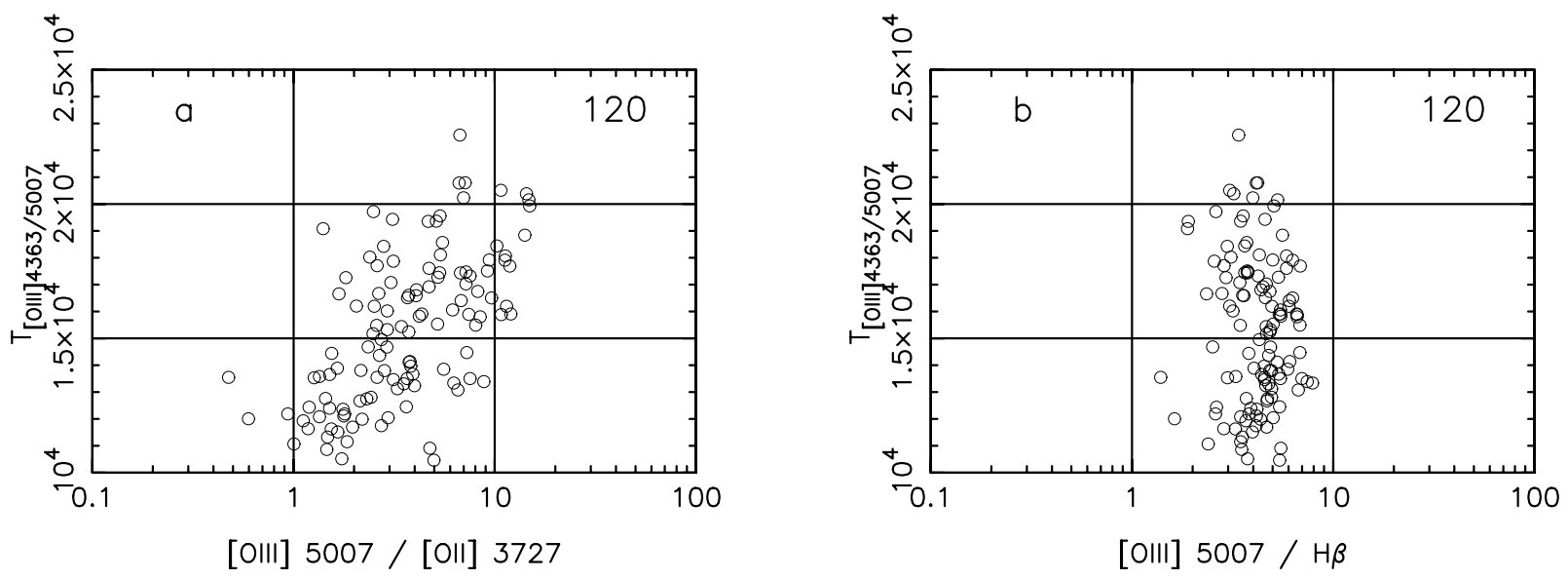

Fig. 3. Our observational sample in the same planes as the models shown in Fig. 1.

Stasińska \& Schaerer 1999; Luridiana et al. 1999; Luridiana \& Peimbert 2001). Reducing the abundances of coolants such as carbon or silicon (on which there are virtually no observational constrains) is insufficient to solve the problem. It seems that an additional heating mechanism is at work, and a popular candidate is shock heating. Whatever may be the origin of the additional heating, this means that simple photoionization models returning a $[\mathrm{O}$ III $] \lambda 5007 /[\mathrm{O}$ II $] \lambda 3727$ in the observed range are likely to have too low an electron temperature, and that those returning a $[\mathrm{O}$ III $] \lambda 4363 /[\mathrm{O}$ III $] \lambda 5007$ comparable with observations have too high a degree of ionization. This means that the amount of collisional excitation they predict for the hydrogen lines is likely a lower limit, since the contribution of collisional excitation increases with electron temperature and with the proportion of residual neutral hydrogen.

\section{Model predictions for the collisional excitation of hydrogen lines}

In Fig. 4, we display the same sequences of models as in Fig. 1, but up to ages of $5 \mathrm{Myr}$, in the $\left.T_{[\mathrm{O}} \mathrm{III}\right] 4363 / 5007$ versus $[\mathrm{O}$ III $] \lambda 5007 / \mathrm{H} \beta$ plane. Again, the top panels correspond to a total initial stellar mass of $M_{\star}=10^{3} M_{\odot}$, the middle ones to $M_{\star}=10^{6} M_{\odot}$ and the bottom ones to $M_{\star}=10^{9} M_{\odot}$. The collisional contribution to the Balmer line emission is indicated in the following way. In the left panels, next to the models are indicated the values of:

$1000 \times\left(\frac{(\mathrm{H} \alpha / \mathrm{H} \beta)(\text { model })}{(\mathrm{H} \alpha / \mathrm{H} \beta)(\text { case B })}-1\right)$

where the case $B$ value of $\mathrm{H} \alpha / \mathrm{H} \beta$ is computed for the temperature $\left.T_{[\mathrm{O}} \mathrm{III}\right] 4363 / 5007$ deduced from the [O III $] \lambda 4363 /[\mathrm{O}$ III $] \lambda 5007$ ratio predicted by the model.

It is seen that in our models the $\mathrm{H} \alpha / \mathrm{H} \beta$ ratios may be larger than the case $\mathrm{B}$ value (at the temperature $\left.T_{[\mathrm{O} \text { III }] 4363 / 5007}\right)$ by up to $8 \%$. This means that the reddening computed assuming the case $\mathrm{B}$ value of the $\mathrm{H} \alpha / \mathrm{H} \beta$ ratios may be significantly overestimated.
In the right panels, next to the models, we indicate (in units of 1 per 1000) the collisional contribution to the emission in the $\mathrm{H} \beta$ line:

$1000 \times\left(\frac{\mathrm{H} \beta(\text { model })}{\mathrm{H} \beta(\text { case } \mathrm{B})}-1\right)$

where $\mathrm{H} \beta$ (case $\mathrm{B}$ ) stands for the integrated emission of the model in the $\mathrm{H} \beta$ line at the local electron temperature.

Clearly, the collisional contribution to $\mathrm{H} \beta$ in our models may reach $2 \%$.

The computations in PHOTO have been made with the collisional excitation rate coefficients from Aggarwal (1983) for the $n=3$ level of hydrogen, and from Drake \& Ulrich (1980) for higher levels. Values given by other authors can be significantly different. For example Aggarwal et al. (1991) give values that are larger than the ones from Drake \& Ulrich by up to a factor 2. Unfortunately, there is so far no agreement on what are the most reliable values of the collision strengths for hydrogen (Callaway 1994).

Note that there is no simple relation between the total $\mathrm{H} \alpha$ and $\mathrm{H} \beta$ emission in our models, although at each point in the nebula the relation between the $\mathrm{H} \alpha$ and $\mathrm{H} \beta$ emissivities is a function of the local temperature only. The reason is that the electron temperature in our models is not uniform, and the relation between local $\mathrm{H} \alpha$ and $\mathrm{H} \beta$ emissivities do not propagate in a simple way into integrated line ratios. It is for a similar reason that a temperature correction factor $t c f$ has been introduced by Sauer \& Jedamzik (2001) to derive the helium abundance from the ratios of helium to hydrogen line intensities.

In real nebulae, where the temperature structure is much more erratic than in simple photoionization models, the situation is probably even more complicated.

One might be tempted to use the ratio of $\mathrm{H}$ Lyman $\alpha$ to $\mathrm{H} \beta$ to better constrain the amount of collisional excitation of hydrogen lines in real nebulae. However, this proves difficult, because the path of $\mathrm{H}$ Lyman $\alpha$ photons is strongly increased by scattering in the ionized as well as in the neutral part of the nebula, during which absorption by dust and molecular hydrogen occur. Therefore, the observed intensity of $\mathrm{H}$ Lyman $\alpha$ strongly depends on the gas and dust distribution and on the velocity field. 

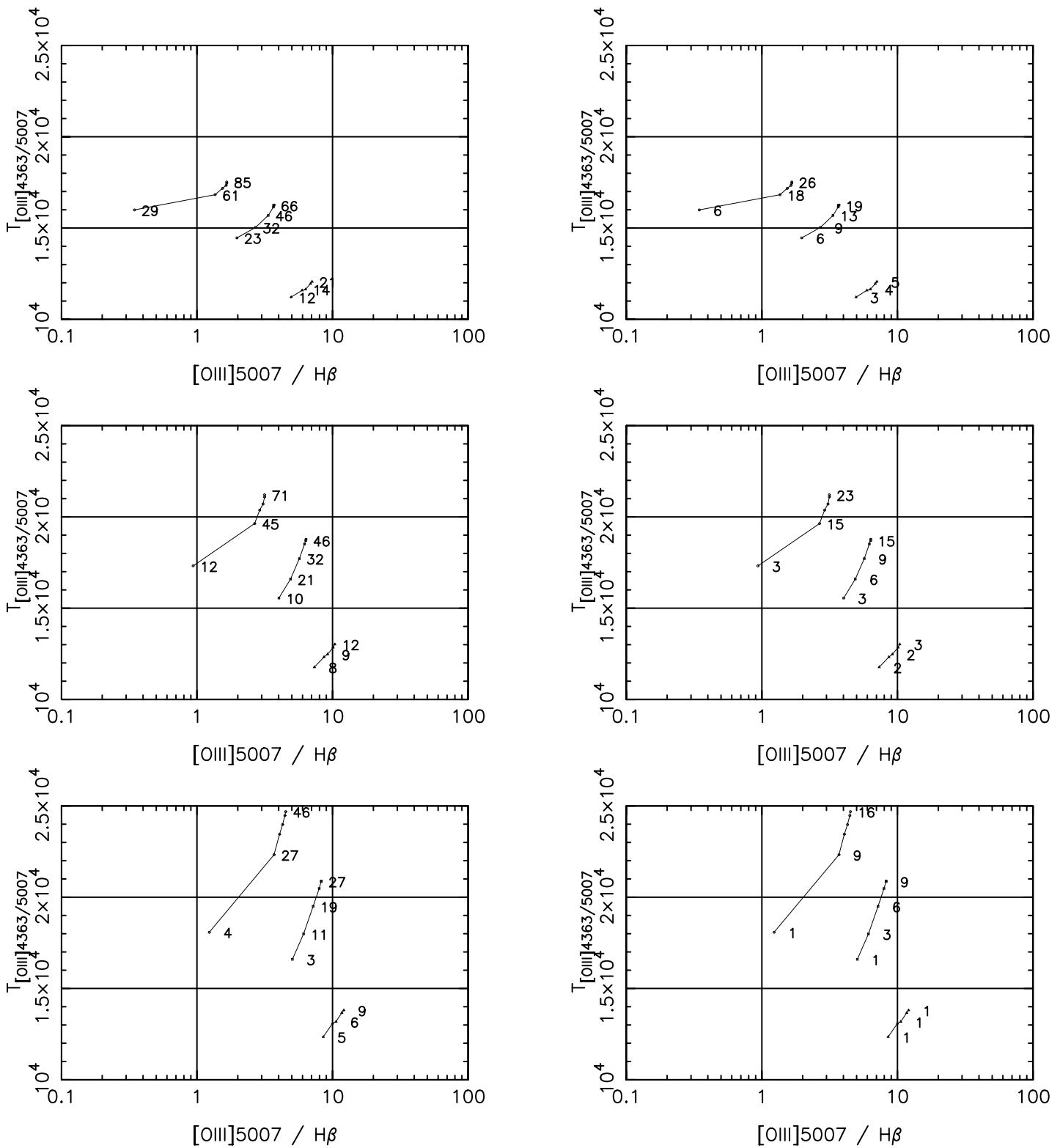

Fig. 4. The same models as in Fig. 1 shown in the $\left.T_{[\mathrm{O}} \mathrm{II}\right] 4363 / 5007$ versus $[\mathrm{O}$ III $] \lambda 5007 / \mathrm{H} \beta$ plane, but only for starburst ages up to 5 Myr. As in Fig. 1, the models shown in the top panels have $M_{\star}=10^{3} M_{\odot}$, those in the middle panels have $M_{\star}=10^{6} M_{\odot}$, those in the bottom panels have $M_{\star}=10^{9} M_{\odot}$. The numbers indicate, in units of 1 per 1000, the values of deviation from case $\mathrm{B}$ theory for $\mathrm{H} \alpha / \mathrm{H} \beta$ (left panels) and $\mathrm{H} \beta$ (right panels). See text for a more detailed explanation.

\section{The effect of $\mathbf{H}$ collisional excitation on the derived helium abundances for a few selected blue compact galaxies}

It is instructive to investigate what are the effects of collisional excitation of the hydrogen lines on the derived helium abundance. This is done in the present section on a few examples.

We have selected 4 low-metallicity blue compact galaxies SBS 0335-052 $\left(Z_{\odot} / 40\right.$, Izotov et al. 2001b), SBS $0940+544\left(Z_{\odot} / 27\right.$, Guseva et al. 2001), Tol 1214-277 and Tol $65\left(Z_{\odot} / 23\right.$, Izotov et al. 2001a) from the sample presented in Sect. 3, on the basis of their high-quality spectra obtained with the Keck telescope and high electron temperatures in the range of $17000-20000 \mathrm{~K}$ derived from the $[\mathrm{O}$ III $]$ line intensity ratios. Because of the high electron temperature the effect of collisional excitation on the helium abundance determination is expected to be relatively large in these galaxies. To derive the ${ }^{4} \mathrm{He}$ and heavy element abundances we follow the prescriptions by Izotov et al. (1994), but assuming that there is a certain percentage of collisional excitation in the $\mathrm{H} \alpha$ and in the $\mathrm{H} \beta$ lines. We assume that the temperature characteristic of the emission of all He I lines is given by $\left.T_{[\mathrm{O}} \mathrm{III}\right] 4363 / 5007$, and that the ionization correction factor for helium is equal to 1 . This is not exactly true for all our models, as seen in Fig. 5, which shows $\left(T_{[\mathrm{O} \text { III }] 4363 / 5007} / T\left(\mathrm{H}^{+}\right)\right.$(left panels) and $\left(x\left(\mathrm{He}^{+}\right)+x\left(\mathrm{He}^{++}\right)\right) / x\left(\mathrm{H}^{+}\right)$(right panels) for the same models as Fig. $4 . T\left(\mathrm{H}^{+}\right)$is defined as

$T_{\mathrm{e}}\left(\mathrm{H}^{+}\right)=\frac{\int T_{\mathrm{e}} n\left(\mathrm{H}^{+}\right) n_{\mathrm{e}} \mathrm{d} V}{\int n\left(\mathrm{H}^{+}\right) n_{\mathrm{e}} \mathrm{d} V}$ 

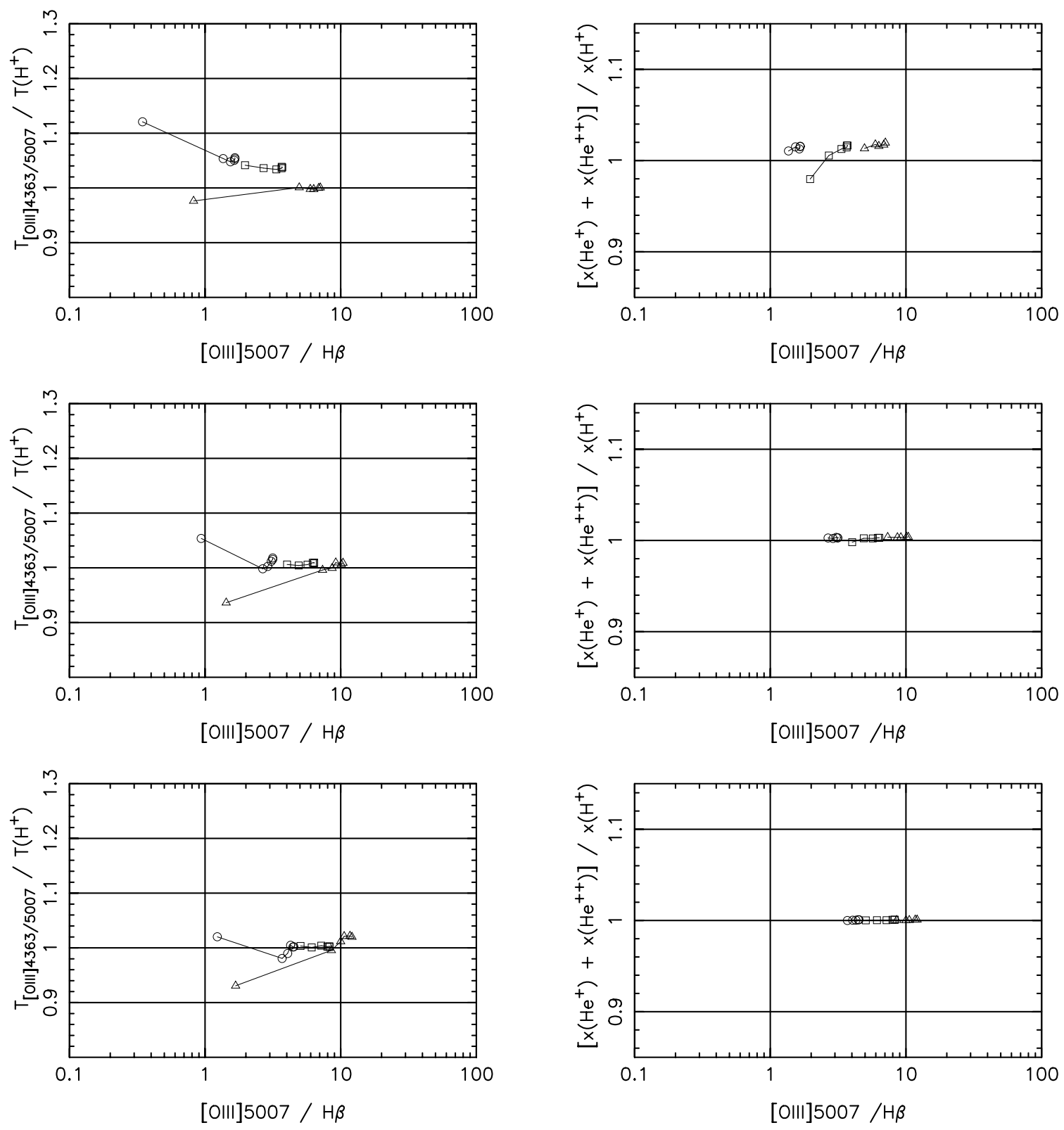

Fig. 5. The same models as in Fig. 4. The symbols have the same meaning as in Fig. 1. As in Figs. 1 and 4 , models shown in the top panels have $M_{\star}=10^{3} M_{\odot}$, those in the middle panels have $M_{\star}=10^{6} M_{\odot}$, those in the bottom panels have $M_{\star}=$ $10^{9} M_{\odot}$. The left panels concern the temperature structure of the models and show the ratio of $\left.T_{[\mathrm{O}} \mathrm{III}\right] 4363 / 5007 / T\left(\mathrm{H}^{+}\right)$versus $[\mathrm{O}$ III $] \lambda 5007 / \mathrm{H} \beta$. The right panels concern the ionization structure of hydrogen and helium and show $\left(x\left(\mathrm{He}^{+}\right)+x\left(\mathrm{H}^{+}\right)\right) / x\left(\mathrm{H}^{+}\right)$ versus $[\mathrm{O}$ III $] \lambda 5007 / \mathrm{H} \beta$.

and $x\left(\mathrm{He}^{+}\right)$is defined as

$x\left(\mathrm{He}^{+}\right)=\frac{\int n\left(\mathrm{He}^{+}\right) n_{\mathrm{e}} \mathrm{d} V}{\int n(\mathrm{He}) n_{\mathrm{e}} \mathrm{d} V}$.

However, since the four considered galaxies have $T_{\text {[O III] } 4363 / 5007}$ around $20000 \mathrm{~K}$ and $[\mathrm{O}$ III $] \lambda 5007 / \mathrm{H} \beta$ larger than 3 , our model grid indicates that our approximations should be reasonable.

In order to explore the effects of collisional excitation of the $\mathrm{H}$ lines, we consider two different cases. In the first case the effect of collisional excitation is accounted for by decreasing by some amount the intensity of the $\mathrm{H} \alpha$ emission line and leaving unchanged the intensity of the $\mathrm{H} \beta$ emission line. In the second case we decrease the intensities of $\mathrm{H} \alpha$ and $\mathrm{H} \beta$ emission lines assuming that the effect of the collisional excitation on the $\mathrm{H} \beta$ line intensity is three times smaller than that on the $\mathrm{H} \alpha$ line intensity. For each assumed value of the contribution of the collisional enhancement to $\mathrm{H} \alpha, \Delta I(\mathrm{H} \alpha) / I(\mathrm{H} \alpha)$, we compute the extinction, correct the emission line ratios for reddening and underlying stellar absorption, then recompute $T_{[\mathrm{O} \text { III]4363/5007 }}$ and derive the ${ }^{4} \mathrm{He}$ and the heavy element abundances. In the analysis, we use the five strongest He I emission lines to derive the helium mass fraction $Y$, after correction for collisional and fluorescent excitation of the He I emission lines. In short, using the intensities of the five He I emission 




Fig. 6. The dependence of the relative correction of the He mass fraction $\Delta Y / Y$ on the contribution of the collisional enhancement $\Delta I(\mathrm{H} \alpha) / I(\mathrm{H} \alpha)$ of the $\mathrm{H} \alpha$ emission line in four low-metallicity blue compact galaxies. The solid line is the dependence when only the intensity of $\mathrm{H} \alpha$ emission line is decreased by the amount indicated by the abscissa. The dotted line shows the dependence when the $\mathrm{H} \alpha$ line intensity is decreased by this amount and the $\mathrm{H} \beta$ line intensity by one third of this amount.

lines, we evaluate the electron number density $n_{\mathrm{e}}$ in the $\mathrm{He}^{+}$zone and the optical depth $\tau(\lambda 3889)$ in the He I $\lambda 3889$ line in a self-consistent way, so that the He I $\lambda 3889 / \lambda 5876$, $\lambda 4471 / \lambda 5876, \lambda 6678 / \lambda 5876$ and $\lambda 7065 / \lambda 5876$ line ratios have their recombination values, after correction for collisional and fluorescent enhancement of the He I emission lines. These effects are very strong in hot low-metallicity $\mathrm{H}$ II regions. In particular, the correction of the He I $\lambda 5876$ emission line for collisional and fluorescent enhancement in SBS $0335-052$ is $\sim 12 \%$.

Of course, for each object, there is a maximum contribution of collisional enhancement $\Delta I(\mathrm{H} \alpha) / I(\mathrm{H} \alpha)$ imposed by the observed $\mathrm{H} \alpha / \mathrm{H} \beta$. In reality, the range of possible values of $\Delta I(\mathrm{H} \alpha) / I(\mathrm{H} \alpha)$ is smaller because interstellar extinction must be present at some level. We have checked that, after the completion of the whole procedure, the higher order Balmer lines remain compatible with case B within observational errors and uncertainties in the extinction law.

In Fig. 6 we show the variations of the correction of the helium mass fraction $\Delta Y / Y$ derived from the He I $\lambda 5876$ emission line for the four galaxies we investigated. It is seen that the correction for the collisional enhancement of the hydrogen lines results in an increase of the He abundance in all galaxies. Qualitatively such a dependence can be explained by two effects: 1 ) the correction of the $\mathrm{H} \beta$ line in the second case (dotted line) increases the $\mathrm{He} \mathrm{I/H} \beta$ intensity ratios; 2) the correction of $\mathrm{H} \alpha$ and $\mathrm{H} \beta$ lines for collisional excitation decreases the extinction and hence increases the intensity of He I $\lambda 5876$ emission line. Certainly, some part of the deviation of the observed $\mathrm{H} \alpha / \mathrm{H} \beta$ intensity ratio from the case $\mathrm{B}$ value is due to interstellar extinction. Therefore, although the effect of collisional excitation of the hydrogen lines on the helium abundance is significant, it is unlikely that the correction $\Delta Y / Y$ exceeds $5 \%$. At larger values of $\Delta Y / Y$ the interstellar extinction derived from the $\mathrm{H} \alpha / \mathrm{H} \beta$ becomes very small, less than the foreground extinction produced in our Galaxy. Our estimate of $\Delta Y / Y$ is not very certain and should be considered as a qualitative indication of the relatively large positive correction of the helium abundance if collisional excitation of the hydrogen emission lines is taken into account. An accurate determination of the helium abundance requires detailed photoionization modeling of the $\mathrm{H}$ II regions in individual galaxies, with all relevant effects taken into account, and with all the relevant line intensities satisfactorily reproduced. Such an enterprise is still ahead of us.

\section{Conclusion and prospects}

Our consideration of the collisional excitation of hydrogen emission lines has shown that this effect can be particularly large, as high as $8 \%$ for the $\mathrm{H} \alpha / \mathrm{H} \beta$ ratio in the lowmetallicity extragalactic $\mathrm{H}$ II regions. If taken into account it results in an upward correction of the helium abundance $Y$ by up to $5 \%$, making the collisional excitation of hydrogen one of the most important sources of systematics in the primordial helium abundance determination. Some other sources of systematic uncertainties should be considered as well. One of the most important sources may be the "temperature fluctuations" which, if present, result in the 
downward correction of $Y$ by $\lesssim 5 \%$ (e.g., Peimbert et al. 2000). Sauer \& Jedamzik (2001) considered the effect of the ionization structure and large-scale temperature variations in the $\mathrm{H}$ II region on the ${ }^{4} \mathrm{He}$ abundance determination. Izotov et al. (2001a) compared the observed properties of the high-excitation $\mathrm{H}$ II regions in the galaxies Tol 1214-277 and Tol 65 with Sauer \& Jedamzik models and found that a small downward $Y$ correction (by less than $1 \%$ ) should be applied due to the two mentioned effects. Finally, underlying stellar He I line absorption of the ionizing clusters should be taken into account. However, this effect is not large in the four considered blue compact galaxies because of the large equivalent widths of emission lines and results in an upward $Y$ correction by $\lesssim 1 \%$. Combining all these systematic effects it appears that the helium abundance derived by Izotov \& Thuan (1998) and Izotov et al. (1999) in low-metallicity blue compact galaxies could be underestimated by about $2-3 \%$ only. However, additional work has to be done to clarify the importance of the systematic effects in the ${ }^{4} \mathrm{He}$ abundance determination. Especially, the mystery of the "temperature fluctuations" has to be solved. For this, a detailed modeling of the $\mathrm{H}$ II regions in each of the best observed low-metallicity blue compact galaxies should be done.

Acknowledgements. Y. I. I. has been partly supported by INTAS 97-0033 grant, and acknowledges support from the "Kiev project" of the Universite Paul Sabatier of Toulouse. He is grateful for the hospitality of the Paris-Meudon Observatory and of the Midi-Pyrenees Observatory where this work was conducted.

\section{References}

Aggarwal, K. M. 1983, MNRAS, 202, 15

Aggarwal, K. M., Berrington, K. A., Burke, P. G., Kingston, A. E., \& Pathak, A. 1991, J. Phys. B: At. Mol. Opt. Phys., 24,1385

Armour, M.-H., Ballantyne, D. R., Ferland, G. J., Karr, J., \& Martin, P. G. 1999, PASP, 111, 1251

Balbes, M. J., Boyd, R. N., \& Mathews, G. J. 1993, ApJ, 418, 229

Ballantyne, D. R., Ferland, G. J., \& Martin, P. G. 2000, ApJ, 536, 773

Benjamin, R. A., Skillman, E. D., \& Smits, D. P. 1999, ApJ, 514,307

Callaway, J. 1994, Atomic Data and Nuclear Data Tables, 57, 9

Campbell, A. 1988, ApJ, 335, 644

Campbell, A. 1992, ApJ, 401, 157

Clegg, R. E. S. 1989, in Planetary Nebulae, ed. S. TorresPeimbert (Dordrecht: Kluwer), IAU Symp., 131, 139

Cota, S. A., \& Ferland, G. J. 1988, ApJ, 326, 889

Davidson, K., \& Kinman, T. D. 1985, ApJS, 58, 321

Dinerstein, H. L., \& Shields, G. A. 1986, ApJ, 311, 45

Drake, S. A., \& Ulrich, R. K. 1980, ApJS, 42, 351

Guseva, N. G., et al. 2001, A\&A, in press, preprint [astro-ph/0109256]

Hummer, D. G., \& Storey, P. J. 1992, MNRAS, 254, 277

Izotov, Y. I., Chaffee, F. H., Foltz, C. B., et al. 1999, ApJ, 527, 757
Izotov, Y. I., Chaffee, F. H., \& Green, R. F. 2001a, ApJ, in press, preprint [astro-ph/0109255]

Izotov, Y. I., Chaffee, F. H., \& Schaerer, D. 2001b, A\&A, in press, preprint [astro-ph/0109254]

Izotov, Y. I., \& Thuan, T. X. 1998, ApJ, 497, 227

Izotov, Y. I., Thuan T. X., \& Lipovetsky, V. A. 1994, ApJ, 435, 647

Izotov,Y. I., Thuan T. X., \& Lipovetsky, V. A. 1997, ApJS, 108, 1

Kunth, D., \& Sargent, W. L. W. 1983, ApJ, 273, 81

Mathews, G. J., Boyd, R. N., \& Fuller, G. M. 1993, ApJ, 403, 65

McGaugh, S. S. 1991, ApJ, 380, 140

Meynet, G., Maeder, A., Schaller, G., Schaerer, D., \& Charbonnel, C. 1994, A\&AS, 103, 97

Luridiana, V., \& Peimbert, M. 2001, ApJ, 553, 633

Luridiana, V., Peimbert, M., \& Leitherer, C. 1999, ApJ, 527, 110

Olive, K. A., \& Skillman, E. D. 2001, New Astron., 6, 119

Olive, K. A., \& Steigman, G. 1995, ApJS, 97, 49

Olive, K. A., Steigman, G., \& Skillman, E. D. 1997, ApJ, 483, 788

Pagel, B. E. J., Simonson, E. A., Terlevich, R. J., \& Edmunds, M. G. 1992, MNRAS, 255, 325

Pagel, B. E. J., Terlevich, R. J., \& Melnick, J. 1986, PASP, 98, 1005

Papaderos, P., et al. 2001, in preparation

Peimbert, M. 1983, in Primordial Helium, ed. P. A. Shaver, D. Kunth, \& Kjär (Garching: ESO), 267

Peimbert, M. 1989, in Elements and the Cosmos, ed. M. G. Edmunds, \& R. J. Terlevich (Cambridge University Press), 196

Peimbert, M., \& Torres-Peimbert, S. 1974, ApJ, 193, 327

Peimbert, M., \& Torres-Peimbert, S. 1976, ApJ, 203, 581

Peimbert, M. Peimbert, A., \& Ruiz, M. T. 2000, ApJ, 541, 688

Peimbert, A. Peimbert, M., \& Luridiana, V. 2001, preprint [astro-ph/0107189]

Rayo, J. F., Peimbert, M., \& Torres-Peimbert, S. 1982, ApJ, 255,1

Sarkar, S. 1996, Rep. Prog. Phys., 59, 1493

Sasselov, D., \& Goldwirth, D. 1995, ApJ, 444, 5

Sauer, D., \& Jedamzik, K. 2001, preprint [astro-ph/0104392]

Schaerer, D., \& de Koter, A. 1997, A\&A, 322, 598

Schaerer, D., \& Vacca, W. D. 1998, ApJ, 497, 618

Schmutz, W., Leitherer, C., \& Gruenwald, R. 1992, PASP, 104, 1164

Shields, G. A. 1986, PASP, 98, 1072

Skillman, E. D., \& Kennicutt, R. C., Jr. 1993, ApJ, 411, 655

Stasińska, G. 1980, A\&A, 84, 320

Stasińska, G. 2001, preprint [astro-ph/0102403]

Stasińska, G., \& Leitherer, C. 1996, ApJS, 107, 661 (SL96)

Stasińska, G., \& Schaerer, D. 1999, A\&A, 351, 72

Stasińska, G., Schaerer, D., \& Leitherer, C. 2001, A\&A, 370, 1 (SSL2001)

Steigman, G., Schramm, D., \& Gunn, J. 1977, Phys. Lett. B, 66,202

Steigman, G., Viegas, S. M., \& Gruenwald, R. 1997, ApJ, 490, 187

Viegas, S. M., Gruenwald, R., \& Steigman, G. 2000, ApJ, 531, 813

Walker, T. P., Steigman, G., Kang, H.-S., Schramm, D. M., \& Olive, K. A. 1991, ApJ, 376, 91 